\title{
Intrinsic eigenvibration frequency in the resonant ultrasound spectroscopy: Evidence for a coupling vibration between a sample and transducers
}

\author{
Akira Yoneda \\ Institute for Study of the Earth's Interior, Okayama University, Misasa, Tottori 682-0193, Japan
}

(Received October 22, 2001; Revised June 4, 2002; Accepted June 6, 2002)

\begin{abstract}
The resonant ultrasound spectroscopy enables us to measure elastic constants of various materials with high accuracy. One of its curious features is resonance frequency shift caused by modulation of clamp force for a sample, although its mechanism has not been clarified yet. A coupling vibration model is newly proposed to interpret this phenomenon and predict its functional form. Further, it is confirmed that the resulted functional forms depend on characteristics of each eigenvibration mode. The extrapolation based on those functional forms enables us to correct measured frequency to the intrinsic one with improved accuracy at least by one order of magnitude. Thus the correction is essential in order to obtain accurate derivatives of frequency with respect to temperature, pressure, composition, and so forth.
\end{abstract}

\section{Introduction}

The resonance method, or resonant ultrasound spectroscopy (RUS), has been extensively used to elucidate elastic properties of various materials (e.g., Sumino et al., 1976; Migliori and Sarrao, 1997; Ohno et al., 2000; Suzuki et al., 2000). One of the main features of the current RUS is that sample must contact to a couple of transducers; one is an oscillator, and the other a receiver for sample vibration (Fig. 1).

It has been recognized that an observed resonance frequency $f$ is slightly shifted with magnitude of clamp-force $F$ to the sample, although its mechanism has not been clarified yet. Therefore intrinsic resonance frequencies, $f_{0}$, have been determined through an empirical extrapolation of $f$ towards $F=0$ (e.g., Sumino et al., 1976), because the current RUS requires a finite magnitude of $F$ in order to transmit vibration energy from transducer to sample, and vice versa.

A coupling vibration between a sample and transducers is inevitably excited on sample-transducer interface. I found that the coupling vibration enables us to explain the magnitude of observed frequency shifts. Then I confirmed that the predicted functional forms explain experimental frequency shifts observed in a sphere sample of soda glass. In this study, I devised a clamp force controller (Appendix A) to increase efficiency and reliability.

I classify vibrational modes of an isotropic sphere into two types according to seismological notation for the earth's free oscillation. While spheroidal modes, ${ }_{q} S_{n}$, are of radial motion, troidal modes, ${ }_{q} T_{n}$, are of no radial motion. The former subscript of $q$ is radial order corresponding number of nodal surface inside the sphere, while the latter angular order corresponding number of nodal lines on the spherical surface. Note that the number of nodal lines on the surface

Copy right(C) The Society of Geomagnetism and Earth, Planetary and Space Sciences (SGEPSS); The Seismological Society of Japan; The Volcanological Society of Japan; The Geodetic Society of Japan; The Japanese Society for Planetary Sciences. is $n$ for ${ }_{q} S_{n}$ modes and $n-1$ for ${ }_{q} T_{n}$ modes.

Note that SI unit is used in this literature except for showing magnitude of clamp force measured in ' $\mathrm{gw}$ '. As $1 \mathrm{gw}=$ $0.0098 \mathrm{~N} \fallingdotseq 0.01 \mathrm{~N}$, it is not difficult to convert ' $\mathrm{gw}$ ' to ' $\mathrm{N}$ ', or vice versa, without losing perspective idea.

\section{Coupling Vibration Model}

We have experienced that the magnitude of $\Delta f / f_{0}$ is of the order of $10^{-3}$ against a few gw of $F$ (see Fig. 2 as an example). Neither deformation of sample nor increase of elastic wave velocities is able to explain such a large deviation of resonance frequency (Appendix B).

Only coupling vibration model (Fig. 3) is able to explain the observed magnitude of frequency shift. The intrinsic angular frequency $\omega_{0}\left(=2 \pi f_{0}\right)$ is defined for the free oscillation state characterized by $k_{s}=0$, or, $F=0$. Thus we have

$$
\omega_{0}^{2}=\frac{2 k}{m}
$$

for the decoupling free, state, where $m$ and $k$ are mass and spring constant, respectively, of the simplified system. Then the effect of weak spring $k_{s}$ is incorporated into the system, we have

$$
\omega^{2}=\frac{2 k+k_{s}}{m}=\omega_{0}^{2}+\frac{k_{s}}{m}
$$

for a coupling state characterized by $k_{s}$, which must be a function of $F$ through an elastic contact between a sample and transducers. This is the essence of the coupling vibration model.

The coupling spring $k_{s}$ is a model of the elastic property at around the contacting region between a sample and transducers; I apply the contacting sphere theory to describe the property (see Fig. 4 and Appendix B).

Although actual eigenvibration modes are not identical with that simplified configuration shown by Fig. 3, we can 
still expect the similar relation under the reasonable assumptions (Appendix C).

According to the contacting sphere theory, the distance between two spheres, $x$, varies with $F$ as

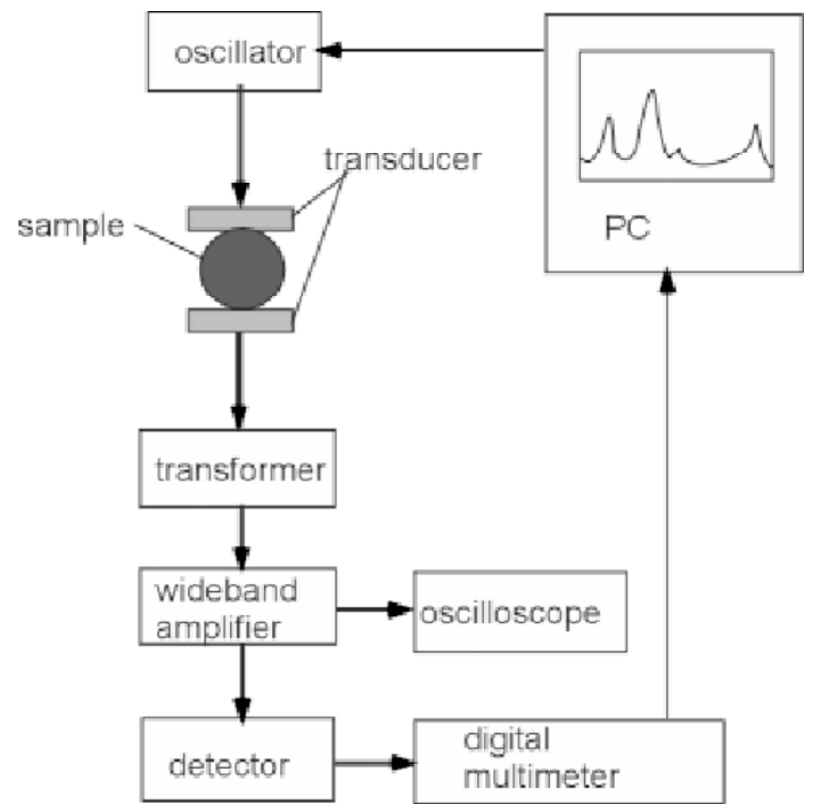

Fig. 1. Block diagram for a RUS experiment. Thick lines correspond to coaxial cable for sinusoidal wave transmission. Thin lines are GPIB cable for digitized data. A set of longitudinal wave transducers (M121 from Panametrics Co.) and shear wave transducers (V156 from Panametrics Co.) are used. Transformer is used to cut low frequency noise.

$$
\Delta x=-g(v, Y, R) F^{2 / 3}
$$

where the factor $g$ is a function of Poisson ratio $v$, Young modulus $Y$, and curvature radius $R$. The area of contact, $A$, is similarly expressed as

$$
A=h(v, Y, R) F^{2 / 3} .
$$

As long as we do not disturb the sample-transducer coupling state, we can assume constant values for $g$ and $h$ during modulation of $F$.

First let consider the case of ${ }_{0} S_{0}$ mode characterized as pure normal displacement of sample surface. In this case, the modeled spring constant, $k_{s}$, is given as

$$
k_{s}=-\frac{d F}{d x}=\frac{3}{2} g^{-1} F^{1 / 3} .
$$

Thus we have the relation:

$$
k_{s} \propto F^{1 / 3} .
$$

Combining (2) and (6), we have a result:

$$
\Delta \omega^{2}=\omega^{2}-\omega_{0}^{2} \propto F^{1 / 3}, \quad \text { or } \quad \Delta f^{2}=f^{2}-f_{0}^{2} \propto F^{1 / 3}
$$

The characteristics of $T$ modes are completely different with those of $S$ modes; $T$ modes are free from normal surface displacement. Therefore, some modification is required for $T$ modes, because the weak spring constant $k_{s}$ corresponds to tangential displacement between sample and transducer. It

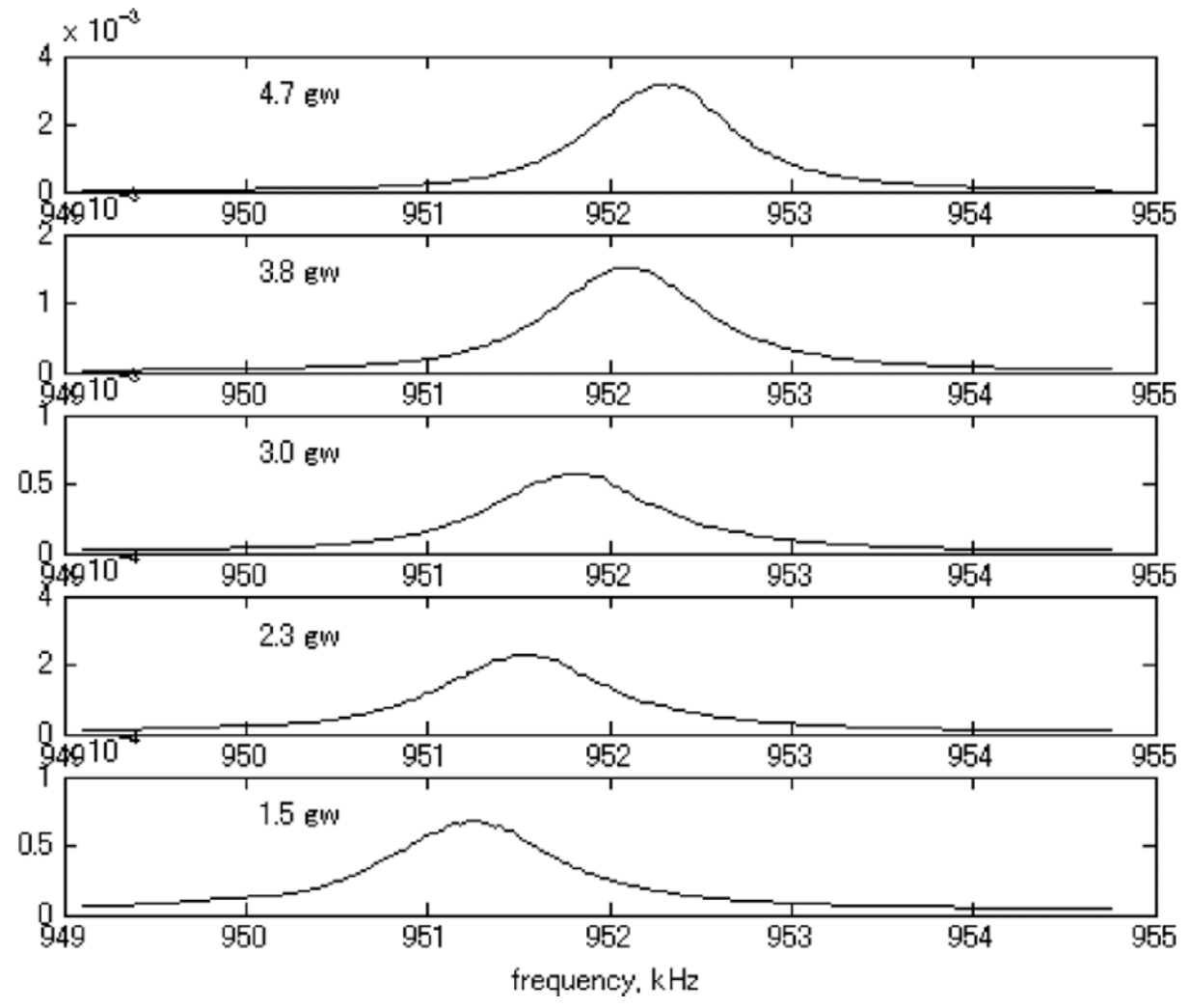

Fig. 2. An example of frequency shift caused by clamp-force modulation; they are ${ }_{0} T_{2}$ peaks of a soda glass sample. The clamp force values are shown in each subplot. We can see that even a few gw clamp force variation causes $\sim 1 \mathrm{kHz}$ peak shift. 

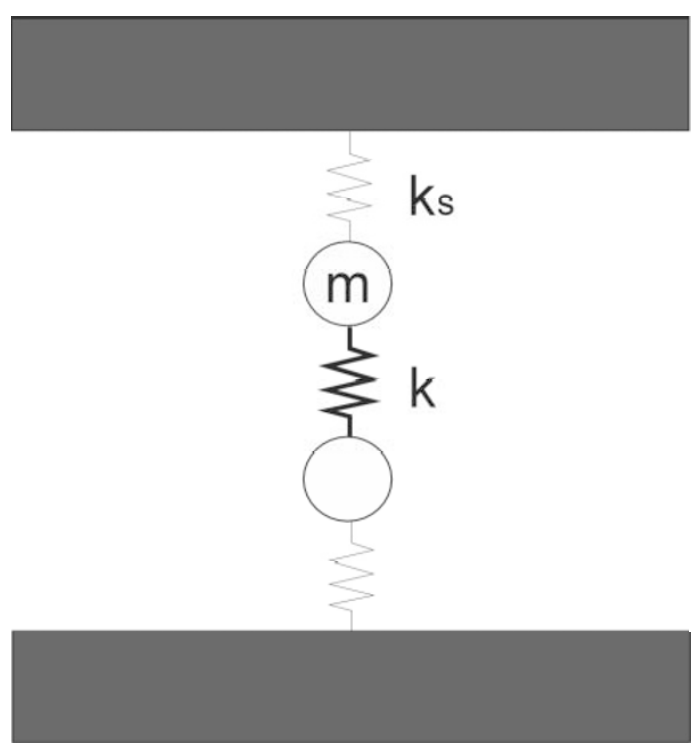

Fig. 3. Illustration for a coupling vibration model. In this figure, an eigen vibration mode is simplified to a harmonic oscillator; the stronger spring constant $k$ corresponds to intrinsic elasticity of sample, and the weaker one $k_{s}$ to extrinsic property at around contact region between sample and transducer. Note that total sample mass is $2 \mathrm{~m}$ in this configuration.

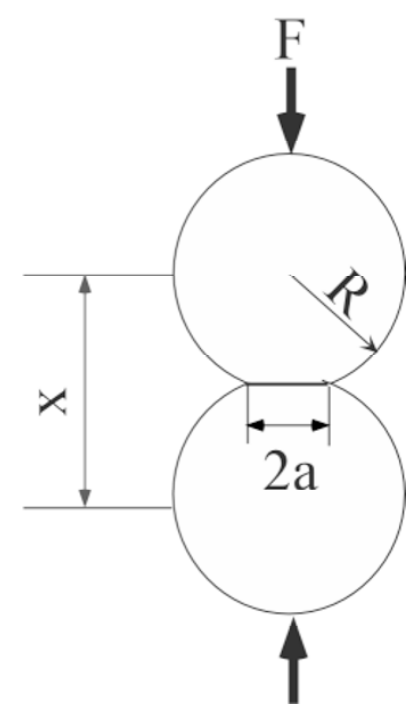

Fig. 4. Illustration for the contacting sphere theory. $R$ is the radius of each sphere. The distance $x$ between the center of the two spheres decreases with $F$. Note that a contacting circle of diameter $2 a$ is formed at the interface of the two sphere owing to their elastic deformation. Therefore, the area of contacting circle, $A$, is $\pi a^{2}$.

may be reasonable to assume $k_{s}$ proportional to the contact area, $A$, between sample and transducer. The contacting sphere theory shows $A$ is in proportion with $F^{2 / 3}$. Therefore we may expect

$$
\Delta \omega^{2} \propto k_{s} \propto F^{2 / 3}
$$

for $T$ modes.

The $S$ modes except ${ }_{0} S_{0}$ are constituted with both normal and tangential surface displacements. Therefore it is difficult to predict the functional form theoretically. It should be revisited after experimental observation.

\section{Experimental}

A soda glass sphere (diameter, $d: 2.890 \mathrm{~mm}$; mass, $m$ : $3.18 \times 10^{-5} \mathrm{~kg}$; density, $\rho: 2.52 \times 10^{3} \mathrm{~kg} / \mathrm{m}^{3}$ ) is used to evaluate the above model, because it is homogeneous and elastically isotropic. In the resonance frequency measurements, clamp force for sample is automatically controlled by a combination of an electric actuator, a load cell, and a voltage regulator (Appendix A). The sample temperature was kept at $25 \pm 0.1^{\circ} \mathrm{C}$ by placing the main part of the system in a temperature-controlled chamber in order to remove a bias caused by temperature fluctuation.

The resonance frequencies measured at various clamp forces are curve-fitted by assuming the proposed functional forms; note that the present correction method does not require any additional information such as physical properties of sample, etc.

\section{Results and Discussion ${ }_{0} S_{0}$ mode}

Figure 5 shows the observed resonance frequency variations observed in two runs separately conducted; we can recognize $\sim 0.5 \mathrm{kHz}$ shift of resonance frequency even at a few gw of $F$. The resulted trends are consistent with the relation (7). Please note that the each trend in Fig. 5 was measured in a single run keeping contacting condition; otherwise, we can recognize that $\Delta f$ may differ even at the same $F$.

The extrapolated free oscillation frequencies converge to 1611.3-1611.4 kHz. In the following discussion, the intrinsic eigenfrequency of ${ }_{0} S_{0}$ is fixed at $1611.4 \mathrm{kHz}$. As a sample is clamped vertically by a couple of transducers, the sample weight may be a possible source of error in $f_{0}$. However its small weight is negligible, because it causes only $\sim 0.02 \mathrm{kHz}$ deviation.

As shown in Appendices B and C, numerical estimation gives theoretical value of $2.2 \times 10^{11} \mathrm{~Hz}^{2} / \mathrm{N}^{1 / 3}$ for $\Delta \omega^{2} / F^{1 / 3}$. The observed gradients in Fig. 5 are calculated to be $2.9 \times$ $10^{11} \mathrm{~Hz}^{2} / \mathrm{N}^{1 / 3}$ and $1.8 \times 10^{11} \mathrm{~Hz}^{2} / \mathrm{N}^{1 / 3}$, respectively, for each run. This agreement in magnitude between theory and experiments is additional support for the coupling vibration model.

\section{$T$ modes}

Resonance frequency variations in $T$ modes $\left({ }_{0} T_{4},{ }_{1} T_{1}\right)$ are shown in Fig. 6; those two modes are selected for comparison, because they have similar resonance frequencies in spite of quite different vibrational character. The main difference is in radial order $q ; q=0$ for ${ }_{0} T_{4}$ and $q=1$ for ${ }_{1} T_{1}$. Although the amplitude information is not presented in Fig. 6, the signal amplitude of $T_{0} T_{4}$ is much larger by $\sim 20$ times than that of ${ }_{1} T_{1}$.

It is impossible to judge which is better model, the $1 / 3$ model or the $2 / 3$ model, just from a first look of Fig. 6 . However we can recognize that the slope of $f^{2}$ variation for ${ }_{1} T_{1}$ is much smaller than that for ${ }_{0} T_{4}$. Note that ${ }_{1} T_{1}$ is characteristic mode having a nodal surface inside, and thus relatively small surface motion. The extrapolated value for ${ }_{1} T_{1}$ is $2190.2 \mathrm{kHz}$ for the $1 / 3$ model and $2190.3 \mathrm{kHz}$ for the $2 / 3$ model; the difference is as small as $0.1 \mathrm{kHz}$.

Note that every $T$ mode frequency is determined only by sample diameter, $d$, and shear wave velocity, $v_{s}$ (e.g., Schreiber et al., 1973). Therefore the resonance frequency of 

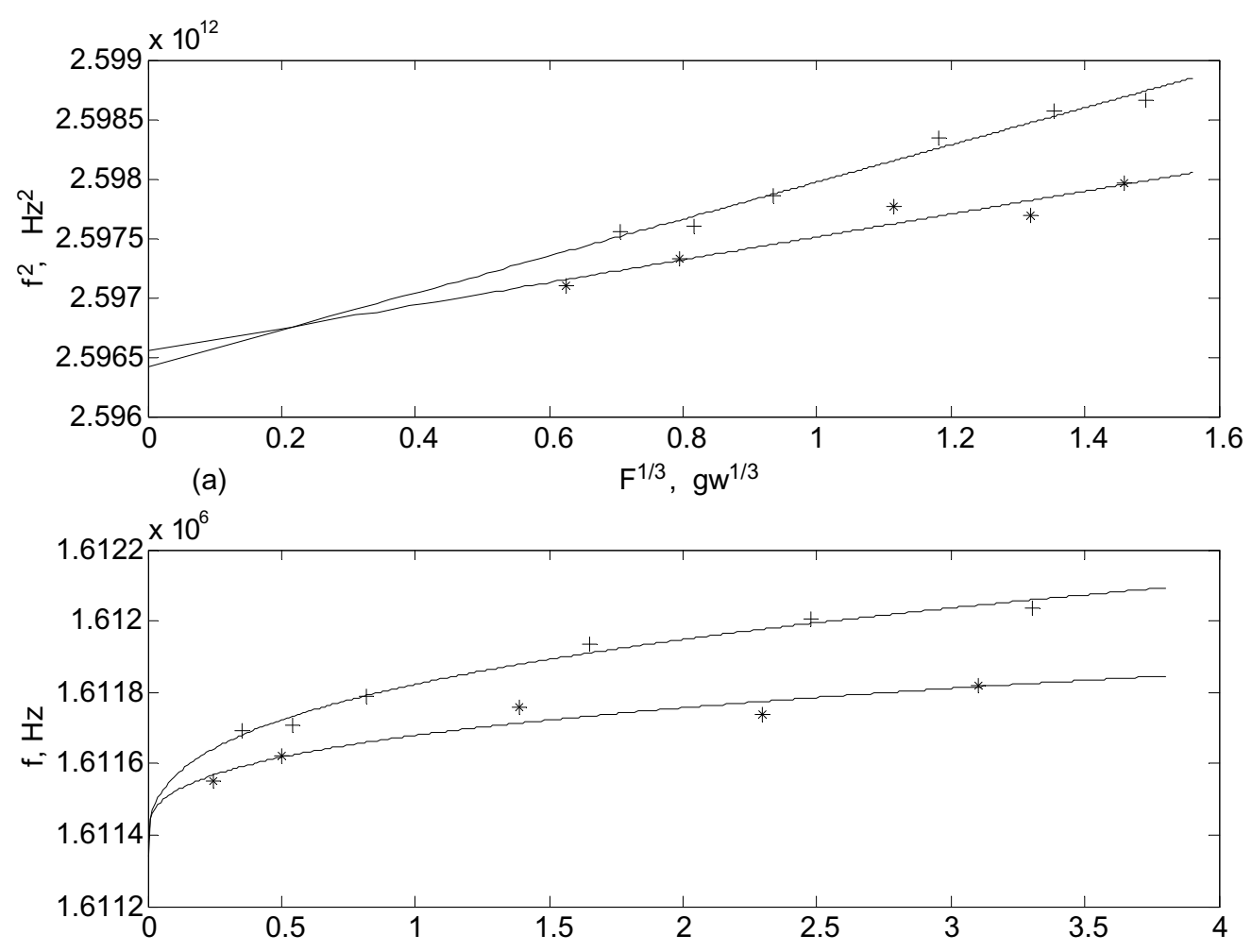

(b)

F, gw

Fig. 5. Frequency shifts for ${ }_{0} S_{0}$ mode in two measurements. Upper frame: $f^{2}-F^{1 / 3}$ plot; Lower frame: $f-F$ plot.
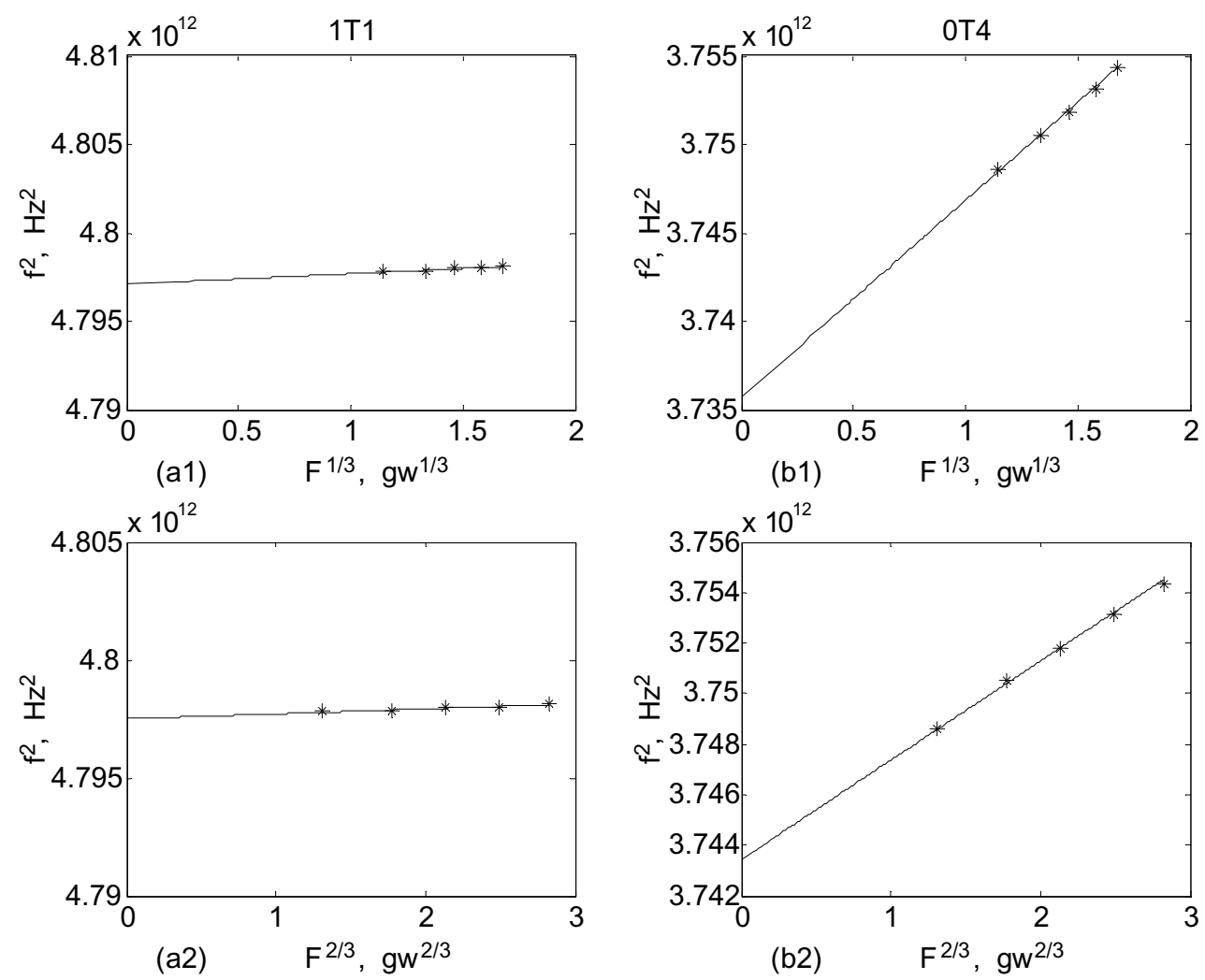

Fig. 6. Frequency shifts for ${ }_{1} T_{1}$ and ${ }_{0} T_{4}$. (a1) $f^{2}-F^{1 / 3}$ plot for ${ }_{1} T_{1}$; (a2) $f^{2}-F^{2 / 3}$ plot for ${ }_{1} T_{1}$; (b1) $f^{2}-F^{1 / 3}$ plot for ${ }_{0} T_{4}$; (b2) $f^{2}-F^{2 / 3}$ plot for ${ }_{0} T_{4}$. 
Table 1. The results for corrected frequencies of $T$ modes. Note that normalized ratio is based on $f_{0}$ of ${ }_{1} T_{1}$ in each column.

\begin{tabular}{cccccc}
\hline Modes & $\begin{array}{c}f_{0} \text { by } \\
\text { the } 1 / 3 \text { model }\end{array}$ & $\begin{array}{c}f_{0} \text { by } \\
\text { the } 2 / 3 \text { model }\end{array}$ & $\begin{array}{c}\text { Normalized ratio in } \\
\text { the } 1 / 3 \text { model }\end{array}$ & $\begin{array}{c}\text { Normalized ratio in } \\
\text { the } 2 / 3 \text { model }\end{array}$ & $\begin{array}{c}\text { Theoretical } \\
\text { ratio }\end{array}$ \\
\hline${ }_{0} T_{2}$ & $948.9 \mathrm{kHz}$ & $950.3 \mathrm{kHz}$ & 0.4333 & 0.4339 & 0.4340 \\
${ }_{0} T_{3}$ & 1466.9 & 1468.2 & 0.6700 & 0.6703 & 0.6706 \\
${ }_{0} T_{4}$ & 1932.9 & 1934.8 & 0.8825 & 0.8834 & 0.8840 \\
${ }_{1} T_{1}$ & 2190.2 & 2190.3 & 1.0 & 1.0 & 1.0 \\
\hline
\end{tabular}

Table 2. The results for corrected frequencies of selected $S$ modes. The theoretically calculated frequencies are also shown in the last column for comparison.

\begin{tabular}{ccccc}
\hline Modes & $\begin{array}{c}f_{0} \text { by } \\
\text { the } 1 / 3 \text { model }\end{array}$ & $\begin{array}{c}f_{0} \text { by } \\
\text { the } 2 / 3 \text { model }\end{array}$ & $\begin{array}{c}\text { Average of the } 1 / 3 \\
\text { and the } 2 / 3 \text { models }\end{array}$ & $\begin{array}{c}f_{0} \text { by theoretical } \\
\text { calculation }\end{array}$ \\
\hline${ }_{0} S_{2}$ & $1001.7 \mathrm{kHz}$ & $1003.0 \mathrm{kHz}$ & $1002.4 \mathrm{kHz}$ & $1002.1 \mathrm{kHz}$ \\
${ }_{1} S_{1}$ & 1282.3 & 1282.8 & 1282.6 & 1282.8 \\
${ }_{1} S_{2}$ & 1823.2 & 1824.1 & 1823.7 & 1824.3 \\
${ }_{0} S_{3}$ & 1483.5 & 1485.6 & 1484.6 & 1484.5 \\
\hline
\end{tabular}

${ }_{1} T_{1}$ can constrain $v_{s}$ most reliably, because its frequency shift is much smaller than that of other $T$ modes. Table 1 summarizes $f_{0}$ ratio with comparing the theoretical one. We can distinguish which is better model from the $f_{0}$ ratio among the $T$ modes. In every case shown in Table 1 , the $2 / 3$ model gives better internal consistency; differences with the theoretical ratios are only a few parts of $10^{-4}$. Therefore I concluded that the $2 / 3$ model is the right model for $T$ modes. Thus the intrinsic eigenfrequency of ${ }_{1} T_{1}$ is fixed at $2190.3 \mathrm{kHz}$.

Now we can obtain longitudinal wave velocity $v_{p}=$ $5811.7 \mathrm{~m} / \mathrm{s}$, shear velocity $v_{s}=3450.4 \mathrm{~m} / \mathrm{s}$, Poisson ratio $v=0.228$, and Young modulus $Y=73.7 \mathrm{GPa}$ from $f_{0}=1611.4 \mathrm{kHz}$ for ${ }_{0} S_{0}, f_{0}=2190.3 \mathrm{kHz}$ for ${ }_{1} T_{1}$, $d=2.890 \mathrm{~mm}$, and $\rho=2.52 \times 10^{3} \mathrm{~kg} / \mathrm{m}^{3}$; these values are used in the following discussion and Appendix B.

$S$ modes except ${ }_{0} S_{0}$

The $S$ modes except ${ }_{0} S_{0}$ are a combination of normal and tangential surface displacement. Therefore it is quite probable that those $f-F$ trends are composite of the $1 / 3$ and the $2 / 3$ models. Table 2 compares experimental results with calculated frequencies based on $v_{p}$ and $v_{s}$ determined in this work.

Comparing those experimental values with calculated ones, we can recognize that the average of the $1 / 3$ and the $2 / 3$ models is consistent with the calculated frequencies. The differences are of only a few parts of $10^{-4}$. Therefore I propose the averaged value for general $S$ modes as a practical and reasonable solution.

The above averaging method may be a kind of compromise; in order to develop more sophisticated correction method, we have to know vibration pattern at the interface between a sample and transducers. Unfortunately it is quite difficult to generate reproducible vibration pattern in the present experimental technique.

\section{Concluding Remarks}

The coupling vibration model clearly explains the frequency shift caused by clamp force modulation. The phenomenon is quite similar to the bond effect recognized in ultrasound velocity measurement (e.g., Yoneda, 1990; Spetzler et al., 1993), because both the problems are originated from vibration energy transfer.

If you just want to obtain elastic constants with accuracy of $10^{-3}$, the resonance frequency correction may be unnecessary. However if you need better accuracy, for instance to obtain precise derivatives of elastic constants on temperature, pressure, or composition, etc., it is very important to apply the present correction method in order to eliminate the ambiguity in resonance frequency.

Last of all, I would like to stress the following three notes in order to enhance accuracy and efficiency:

(1) ${ }_{0} S_{0}$ and $T$ modes are special modes realized only for elastically isotropic sphere sample. If the sample shape is not sphere or physical property of sample in not isotropic, eigenviblation modes are basically combination of normal and tangential surface displacement. Therefore the average of $1 / 3$ and $2 / 3$ models should be recommended in general in order to conduct resonance frequency correction.

(2) Sample and transducers should be clean enough. If the surface is contaminated by greasy substance, you may observe substantial frequency shifts even at negative clamp force. It is not surprising, because "grease" can keep substantial contact between a sample and transducers against tensional force.

(3) As far as my experience, larger amplitude of eigenfrequency peaks tends to have larger frequency shift; an example is amplitude contrast between ${ }_{1} T_{1}$ and ${ }_{0} T_{4}$. It 
is reasonable, because harder coupling between sample and transducer may give simultaneously larger amplitude and larger frequency shift. Therefore smaller amplitude modes may be more important in order to constrain sample properties in spite of relative difficulty in determining the resonant frequency.

Acknowledgments. The author is indebted to Mr. Maeda for designing and assembling the electronic circuit for clamp-force controller. He also thanks Prof. I. Suzuki at Okayama Univ. and Prof. I. Ohno at Ehime Univ. for helpful comments to the present model. This work was partially supported by a Grant-in Aid for Scientific Research No. 12440121 from the Japan Society for the Promotion of Science.

\section{Appendix A. Automatic Clamp-Force Controller}

So far the clamp force has been controlled by manual adjusting of weight on a balance equipped in the resonance measurement system. This is not only inconvenient but also inaccurate due to frictional bias in a balance. Note that the uncertainty of an ordinary commercial balance is as large as $0.2 \mathrm{gw}$, which may cause trouble in the present frequency correction.

Therefore I devised an automatic clamp force controller free from frictional error. Figure A1 shows the concept of the instrument. Figure A2 shows the stability of $F$, or output of load-cell. We can see that $F$ is controlled automatically within $0.1 \mathrm{gw}$, or $\sim 0.001 \mathrm{~N}$, both for long-term variation and short period fluctuation.

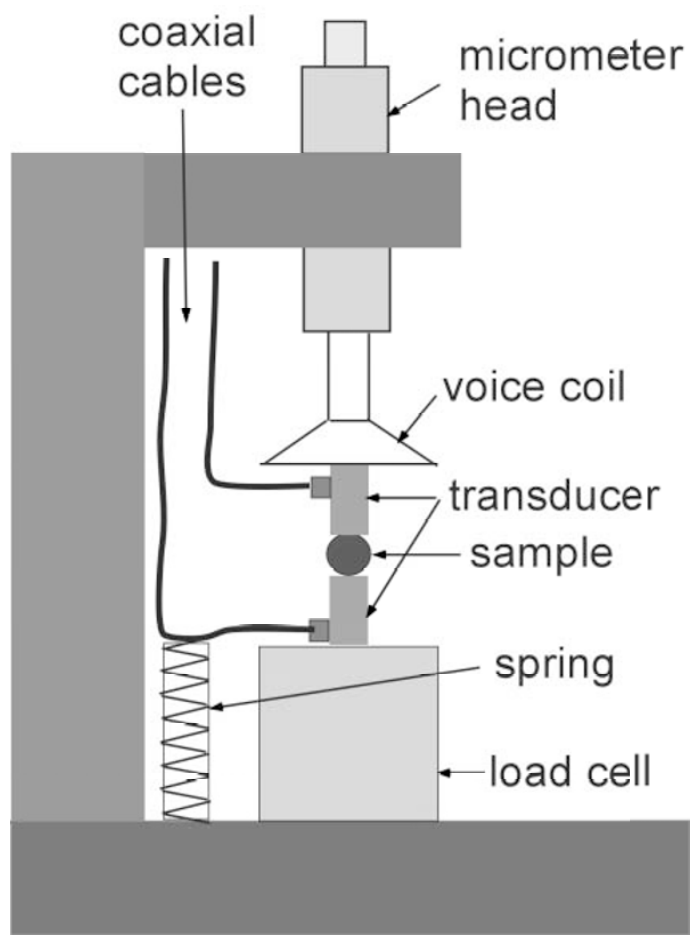

Fig. A1. Schematic diagram of the clamp-force controller. The voice coil is a clamp-force stabilizing actuator; it is operated by a feedback DC current supplier minimizing the difference between the input target voltage and the load cell output. The spring is used to minimize weight bias caused by coaxial cable for transducer.

\section{Appendix B. Contacting Sphere Theory and its Characteristics}

The contacting sphere theory itself is found in many text book (e.g., Timoshenko and Goodier, 1970). It is well known that the theory is applicable as well for sphere-plane contact by assuming infinitely large curvature radius to the plane. Note that sample and transducer are assigned by subsuffixes, 1 and 2, respectively. Elastic properties of the sample are $v_{1}=0.228$ and $Y_{1}=73.8 \mathrm{GPa}$. Its curvature radius, $R_{1}$, is identical with its radius $r_{0}$; thus $R_{1}=r_{0}=d / 2=$ $1.445 \mathrm{~mm}$. Note that the transducers' surface is covered by a thin tungsten-carbide plate $\left(v_{2}=0.22 ; Y_{2}=534 \mathrm{GPa}\right.$; $\left.R_{2}=\infty\right)$.

By substituting those values, the contacting radius $a$ is given as

$$
a=\sqrt[3]{\frac{3 \pi\left(k_{1}+k_{2}\right) R_{1}}{4}} F^{1 / 3} \approx 2.5 \times 10^{-5} F^{1 / 3}
$$

where $F$ is force, and $k_{1}$ and $k_{2}$ are defined as

$$
k_{i}=\frac{1-v_{i}^{2}}{\pi Y_{i}}, \quad i=1,2 .
$$

Then $d x$, the normal displacement between sample and transducer, is given as

$$
d x=\sqrt[3]{\frac{9 \pi^{2}\left(k_{1}+k_{2}\right)^{2}}{16 R_{1}}} F^{2 / 3} \approx 4.4 \times 10^{-7} F^{2 / 3} .
$$

Then we have $k_{s}$ as

$$
k_{s}=\left(\frac{\partial F}{\partial x}\right)=\frac{3 F^{1 / 3}}{2 \times\left(4.4 \times 10^{-7}\right)} \approx 3.4 \times 10^{6} F^{1 / 3} .
$$

In Fig. B1, I present variations of $a, \mathrm{~A}\left(=\pi a^{2}\right), d x$, and $k_{s}$ with $F$ in the present experimental configuration. These parameters are useful to evaluate effect of other possible mechanisms for resonance frequency shift. First, length change $\Delta x / x$ is estimated to be $\sim 10^{-5}$ at 5 gw for a soda glass sample of $\sim 3 \mathrm{~mm}$ diameter. Second, mean pressure at contact area is estimated to be $10^{6} \mathrm{~Pa}$ at $5 \mathrm{gw}$ as well. We can assume that $\Delta v / v \sim \Delta p / C$, where $v$ is elastic wave velocity, $\Delta p$ pressure increment, and $C$ a representative elastic constant $\sim 100 \mathrm{GPa}$. Then substitute $\Delta p=10^{6} \mathrm{~Pa}$, we have $\Delta v / v \sim 10^{-5}$. Note that relative resonance frequency shift $\Delta f / f$ is given by $\Delta x / x+\Delta v / v$. Therefore even the combination of the two mechanisms cannot explain the observed frequency shift of order of $\Delta f / f \sim 10^{-3}$.

\section{Appendix C. Coupling Vibration for a General Eigenvibration Mode}

Actual eigen vibration of sample is much more complicated than the simplified model shown in Fig. 3. Therefore I show here that the coupling vibrational model is still reasonable to any eigenvibration mode. Let suppose the following conditions for eigenvibration modes and the coupling vibration.

(I) The modeled spring $k_{s}$ yields only small perturbation for an eigenvibration mode.

(II) It does not have its own mass like an ideal spring, or cannot store any kinetic energy. 

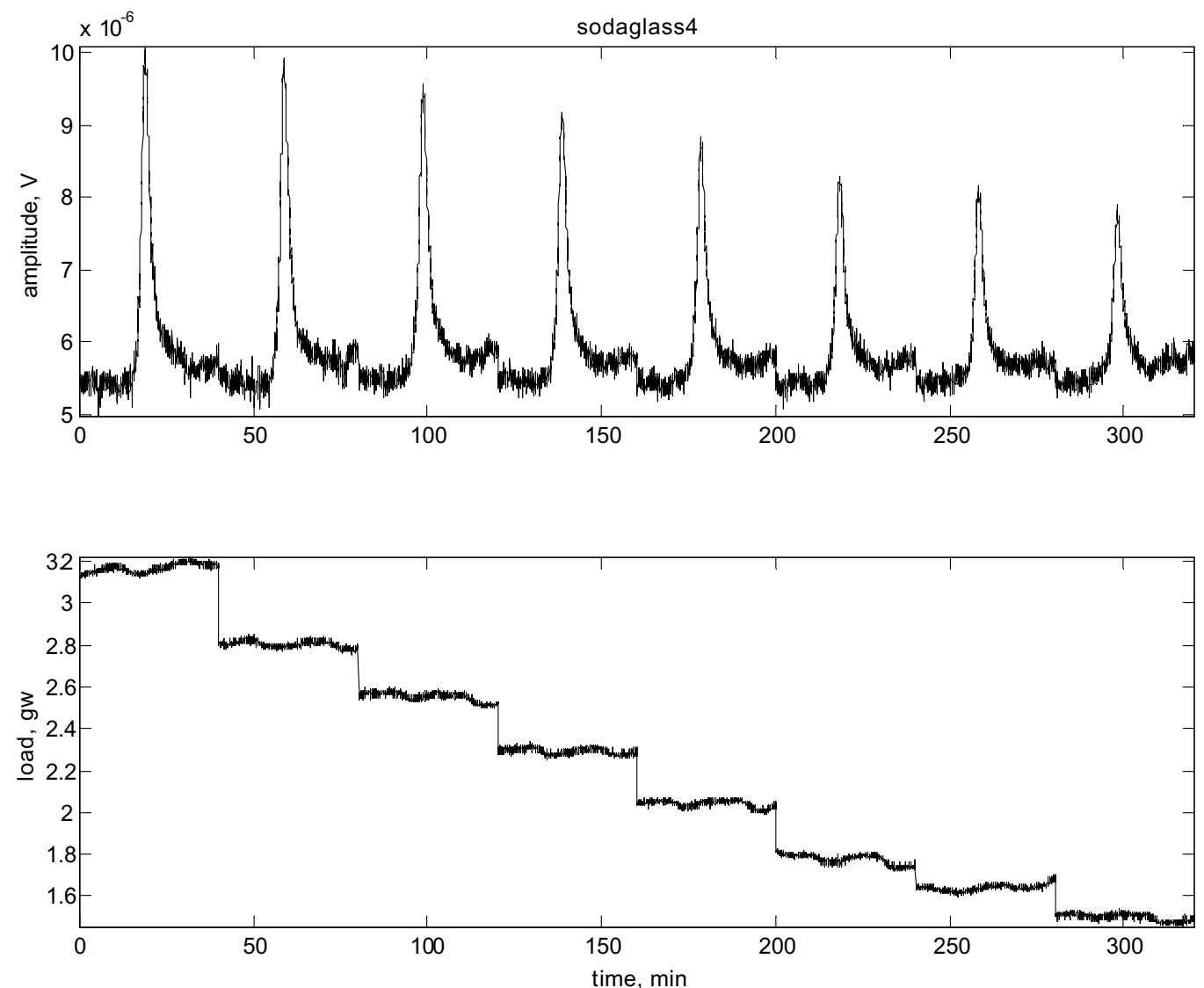

Fig. A2. An example of resonance signals by using the clamp-force controller. We can see that the clamp force is controlled within 0.1 gw. Upper frame: Resonance signals. Lower frame: Load, or clamp force $F$, during the measurement. We can see that amplitude of resonance peaks decreases with decreasing $F$.
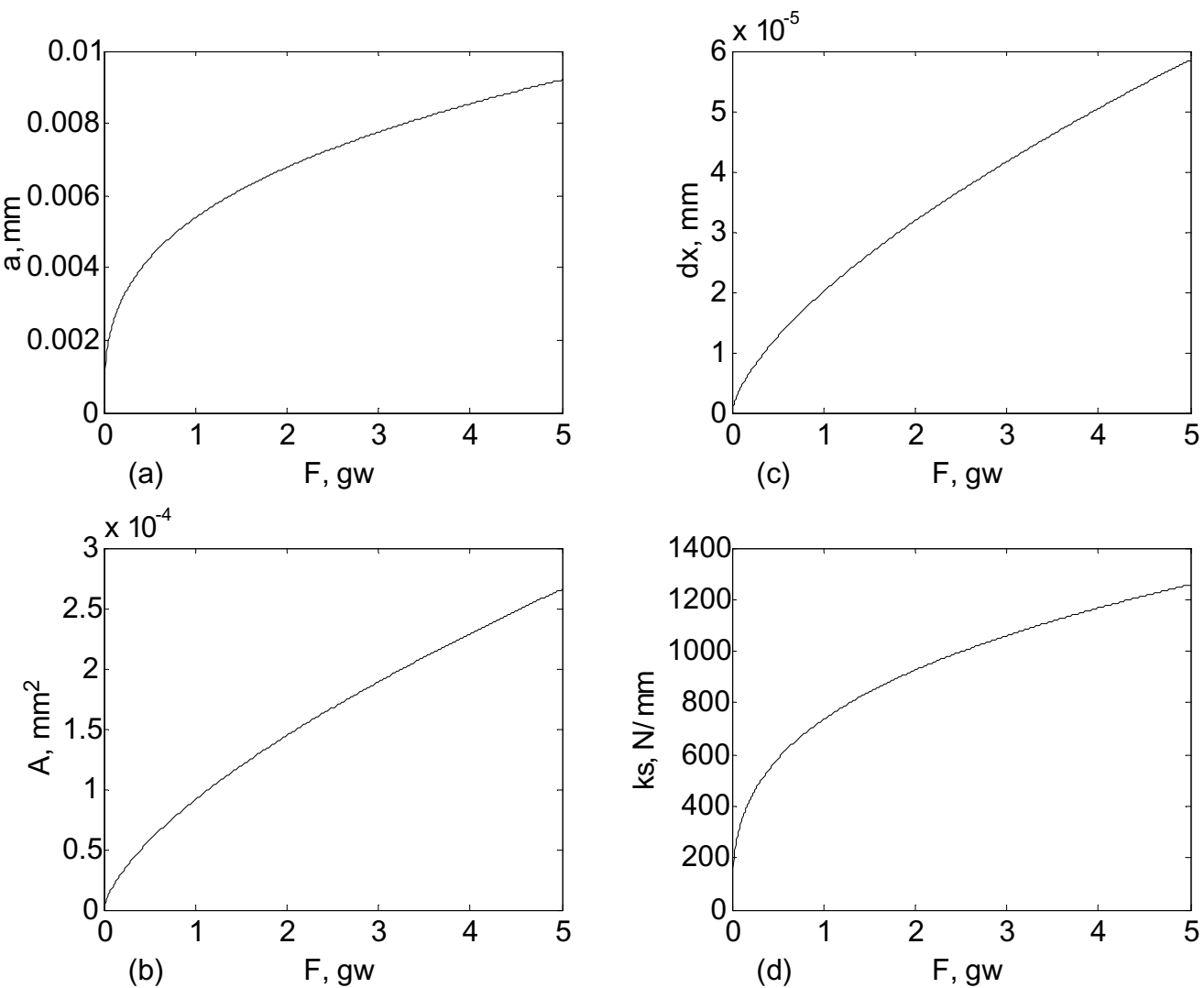

Fig. B1. Useful parameters in the coupling model as a function of clamp force $F$. (a) radius of contacting circle, $a$; (b) area of contacting circle, $A$; (c) displacement of sphere, $d x$; (d) spring constant, $k_{s}$. 
According to the eigenvibration analysis (e.g., Ohno, 1976; Visscher et al., 1991; Yoneda, 2000) intrinsic angular frequency must satisfy

$$
E_{k}=\omega_{0}^{2} \int \frac{1}{2} \rho u_{i} u_{i} d V=\int \frac{1}{2} C_{i j k l} \varepsilon_{i j} \varepsilon_{k l} d V=E_{S}
$$

where $E_{K}$ and $E_{S}$ are kinetic energy and strain energy, respectively, $\rho$ density, $u$ displacement, $C$ elastic stiffness constants, $\varepsilon$ strain. The assumption (I) allows us to modify (C.1) as

$$
\begin{aligned}
\omega^{2} \int \frac{1}{2} \rho u_{i} u_{i} d V= & \int \frac{1}{2} C_{i j k l} \varepsilon_{i j} \varepsilon_{k l} d V+\frac{1}{2} k_{s 1} \delta\left(u_{1}\right)^{2} \\
& +\frac{1}{2} k_{s 2} \delta\left(u_{2}\right)^{2}
\end{aligned}
$$

where $\delta(u)$ is an effective displacement for spring $k_{s}$, and sub-suffixes, 1 and 2, specifies contact states against the two transducers. Because $\delta\left(u_{1}\right) / \delta\left(u_{2}\right)$ is considered to be a constant in each eigenvibration mode, we can combine the additional terms in (C.2) as

$$
\frac{1}{2} k_{s 1} \delta\left(u_{1}\right)^{2}+\frac{1}{2} k_{s 2} \delta\left(u_{2}\right)^{2}=k_{s} \delta(u)^{2} .
$$

Note that there is no additional term for kinetic energy because of assumption (II). Combining (C.1), (C.2), and (C.3), we can derive

$$
\Delta \omega^{2}=\omega^{2}-\omega_{0}^{2} \propto k_{s}
$$

for any eigenvibration mode. Therefore we can apply the coupling vibration model in general.

Last of all, let estimate $\Delta \omega^{2} / F^{1 / 3}$ for ${ }_{0} S_{0}$. In the ${ }_{0} S_{0}$ mode, the surface displacements $u$ is simply expressed in the spherical coordinates:

$$
u_{r}=-\frac{h J_{1.5}(h r)}{\sqrt{r}}, \quad u_{\theta}=u_{\phi}=0
$$

where $h\left(=\omega / v_{p}\right)$ is wave number. Then substituting (C.5) and sample's parameters $\left(\rho=2.52 \times 10^{3} \mathrm{~kg} / \mathrm{m}^{3} ; r_{0}=d / 2=\right.$
$1.445 \mathrm{~mm}$ ) into the following equation

$$
\Delta \omega^{2} \int \frac{1}{2} \rho u_{r}^{2} d V=k_{s} \delta(u)^{2}=k_{s} u_{r}\left(r_{0}\right)^{2}
$$

we have $\Delta \omega^{2} / k_{s}=6.5 \times 10^{4}$. Combining (B.4) and (C.6), we have

$$
\Delta \omega^{2} \approx 2.2 \times 10^{11} F^{1 / 3} .
$$

Note that the above estimation is only possible for ${ }_{0} S_{0}$, because it is the unique mode of uniform surface displacement, $u_{r}\left(r_{0}\right)$.

\section{References}

Migliori, A. and J. Sarrao, Resonant ultrasound spectroscopy, John Wiley \& Sons, New York, 1997.

Ohno, I., Free vibration of a rectangular parallelepiped crystal and its application to determination of elastic constants of orthorhombic crystals, $J$. Phys. Earth, 24, 355-379, 1976.

Ohno, I., M. Abe, M. Kimura, Y. Hanayama, H. Oda, and I. Suzuki, Elasticity measurement of silica glass under gas pressure, American Mineralogist, 85, 288-291, 2000.

Schreiber, E., O. L. Anderson, and N. Soga, Elastic constants and their measurement, McGraw Hill, New York, 1973.

Spetzler, H. A., G. Chen, S. Whitehead, and I. C. Getting, A new ultrasonic interferometry for the determination of equation of state parameter of sub-milimeter single crystal, Pure Appl. Geophys., 141, 341-377, 1993.

Sumino, Y., I. Ohno, T. Goto, and M. Kumazawa, Measurement of elastic constants and internal frictions on single-crystal $\mathrm{MgO}$ by rectangular parallelepiped resonance, J. Phys. Earth, 24, 263-273, 1976.

Suzuki, I., I. Ohno, and O. L. Anderson, Harmonic and anharmonic properties of spinel $\mathrm{MgAl}_{2} \mathrm{O}_{4}$, American Mineralogist, 85, 304-311, 2000.

Timoshenko, S. P. and J. N. Goodier, Theory of Elasticity third edition, p. 412, McGraw Hill, New York, 1970.

Visscher, W. M., A. Migliori, T. M. Bell, and R. A. Reinert, On the normal modes of free vibration of inhomogeneous and anisotropic elastic objects, J. Acoust. Soc. Am., 90, 2154-2162, 1991.

Yoneda, A., Pressure derivatives of elastic constants of single crystal MgO and $\mathrm{MgAl}_{2} \mathrm{O}_{4}$, J. Phys. Earth, 38, 19-55, 1990.

Yoneda, A., The xyzr algorithm specialized for eigenvibration problem of bored and laminated objects, J. Sound and Vibration, 236, 431-441, 2000 .

A. Yoneda (e-mail: yoneda@misasa.okayama-u.ac.jp) 